\title{
Evaluation of Different Methods for Detection of Metallo-beta-Lactamases in Pseudomonas aeruginosa Clinical Isolates
}

\author{
TOMASZ BOGIEL*, ALEKSANDER DEPTUŁA and EUGENIA GOSPODAREK \\ Department of Microbiology, Nicolaus Copernicus University in Toruń, Collegium Medicum in Bydgoszcz, \\ 9 M. Skłodowska-Curie St, 85-094 Bydgoszcz, Poland
}

Received 30 May 2009, revised 23 November 2009, accepted 1 December 2009

\begin{abstract}
Metallo-beta-lactamases (MBLs) produced by Pseudomonas aeruginosa are a serious threat due to their ability to be transmitted between the same as well as different bacterial species. Different methods are applied in the clinical laboratory to detect MBLs. The aim of this study was to compare 4 phenotypic methods and a PCR assay for their ability to detect MBLs in clinical isolates of carbapenem-resistant $P$. aeruginosa strains. The study embraced a total of 70 carbapenem-resistant $P$. aeruginosa strains isolated in The Department of Microbiology of Dr A. Jurasz University Hospital in Bydgoszcz. The highest percentage (42.9\%) of the strains were isolated from Intensive Care Unit patients, mainly from urine samples (31.4\%). Methods used in this study were: double-disc synergy tests in two combinations: using ceftazidime with 2-mercaptopropionic acid and imipenem with EDTA, differences in inhibition zone diameters between discs with imipenem/ EDTA and imipenem, Etest MBL (AB Biodisk) and molecular amplification of $b l a_{\mathrm{IMP}}$ and $b l a_{\mathrm{VIM}}$ genes responsible for producing MBLs, using PCR assay. The lowest percentage (1.4\%) of positive results in detection of MBLs was obtained using PCR assay, the highest (72.9\%) by double-disc synergy tests with imipenem and EDTA, but the specificity of this method may be low.
\end{abstract}

K e y w ord s: Pseudomonas aeruginosa, carbapenem resistance, mechanism of resistance, metallo-beta-lactamases (MBLs)

\section{Introduction}

The detection of antimicrobial resistance mechanisms is strictly related to finding appropriate antimicrobial treatment. Since 2003, Annual Recommendations of The National Reference Centre for Antimicrobial Susceptibility Testing (Hryniewicz et al., 2006) contain information regarding resistance to beta-lactams due to synthesis of carbapenemases, such as metallo-beta-lactamases (MBLs). A number of methods for the detection of this kind of enzymes have been described in the literature, because genes responsible for this resistance may be transferred both horizontally and vertically (Fiett et al., 2006). MBLsproducing strains have been increasingly recognized worldwide and different laboratory methods to detect the appropriate enzymes have been developed. Techniques for the detection of MBLs differ from one other not only in cost, but also in the most important properties from the microbiological point of view: specificity and sensitivity.
The aim of this study was to evaluate the utility of different methods for the detection of MBLs in clinical isolates of Pseudomonas aeruginosa.

\section{Experimental}

\section{Material and Methods}

Bacterial strains. The study involved a total of 70 clinical isolates of carbapenem-resistant P. aeruginosa strains isolated from clinical specimens in The Department of Microbiology of Dr A. Jurasz University Hospital in Bydgoszcz, Poland. The bacteria were isolated from patients from different wards - the highest number from the patients of the Intensive Care Unit and the Rehabilitation Clinic, 30 (42.9\%) and $8(11.4 \%)$ strains, respectively (Table I). Out of 70 carbapenem-resistant $P$. aeruginosa strains, 22 (34.1\%) were isolated from urine, from both - wound swabs and tracheotomy tubes - $11(15.7 \%), 6(8.6 \%)$ from

* Corresponding author: T. Bogiel, 9 M. Skłodowska-Curie St, 85-094 Bydgoszcz, Poland; phone: (+48) 52 5853673; fax: (+48) 52 5854047; e-mail: bogiel.tomasz@wp.pl 
Table I

Origin of examined Pseudomonas aeruginosa strains $(\mathrm{n}=70)$

\begin{tabular}{|l|c|r|}
\hline \multicolumn{1}{|c|}{ Ward } & $\begin{array}{c}\text { Number of } \\
\text { examined } \\
\text { strains }\end{array}$ & $\%$ \\
\hline Intensive Care Unit & 30 & 42.9 \\
\hline Rehabilitation Clinic & 8 & 11.4 \\
\hline General Surgery and Endocrinology & 5 & 7.1 \\
\hline Neurosurgery nad Neurotraumatology & 5 & 7.1 \\
\hline General and Vascular Surgery & 3 & 4.3 \\
\hline General, Oncology and Pediatric Urology & 3 & 4.3 \\
\hline Pediatric, Hematology and Oncology & 2 & 2.9 \\
\hline Transplantology and General Surgery & 2 & 2.9 \\
\hline Other Procedural Clinics & 7 & 10.0 \\
\hline Outpatients & 5 & 7.1 \\
\hline
\end{tabular}

bronchoalveolar lavage samples, 5 (7.1\%) from bedsore swabs and 15 (21.4\%) from other specimens.

Species identification. P. aeruginosa identification was done by observation of colony morphology on Columbia Agar with 5\% sheep blood (bioMérieux), ability to grow and pigments production on medium with cetrimide (PseudoSel Agar, Becton-Dickinson), oxidase activity and was confirmed using ID 32 GN (bioMérieux) tests. Tests were made according to the manufacturer's instructions and executed by ATB Expression V 2.8.8 (bioMérieux).

Antimicrobial susceptibility. Antibiograms were performed with disc-diffusion method on Mueller-Hinton Agar (Mueller-Hinton II Agar, Becton-Dickinson) according to Recomendations of The National Reference Centre for Antimicrobial Susceptibility Testing and Clinical and Laboratory Standards Institute (CLSI, 2007) interpretation. As a control $P$. aeruginosa ATCC 27853 was used.

Three $P$. aeruginosa strains with confirmed ability to produce MBLs (IMP- and VIM-like) obtained from Dr Marek Gniadkowski from The Departament of Molecular Microbiology of The National Medicines Institute and PAO1 strain served as positive and negative controls in the detection of MBLs, respectively.

Phenotypic methods for MBLs detection. Four different methods were used to detect MBLs:

1. double disc synergy test using ceftazidime (CAZ $30 \mu \mathrm{g}$, Becton Dickinson) and sterile disc (Blank paper discs $6 \mathrm{~mm}$, Becton-Dickinson) with $4 \mu \mathrm{l}$ of 2-mercaptopropionic acid (2-MPA, Fluka) described by Arakawa et al. (2000),

2. comparison of inhibition zones diameters between imipenem with EDTA (IPM/EDTA, 10/1000 $\mu \mathrm{g}$, Becton Dickinson/Sigma) and imipenem (IPM $10 \mu \mathrm{g}$, Becton Dickinson) according to Yong et al. (2002),

3. double disc synergy test using imipenem (IPM $10 \mu \mathrm{g}$, Becton Dickinson) and sterile disc (Blank paper discs $6 \mathrm{~mm}$, Becton-Dickinson) with $10 \mu \mathrm{l}$ of
0.5 M EDTA solution (Ethylene Diamino Tetraacetic

Acid, Sigma), as described by Lee et al. (2003),

4. Etest MBL (AB Biodisk) comparing MIC values between imipenem (IPM) and imipenem with EDTA (IPI).

In the case of each phenotypic method, several colonies from a 24-hour culture plate were used to prepare a $0.5 \mathrm{McF}$ arland density inoculum in $0.85 \% \mathrm{NaCl}$ solution. Mueller-Hinton Agar (Becton-Dickinson) plates were streaked using cotton swabs and appropriate discs or Etests strips were then applied, respectively. The plates were incubated for $16-20$ hours at $35^{\circ} \mathrm{C}$.

Interpretation of phenotypic methods for detection of MBLs. An observation of the deformed inhibition zones in the case of any antibiotic disc or at least $7 \mathrm{~mm}$ of zone size enhancements with EDTA impregnated imipenem discs were interpreted as positive results for combinations of double disc synergy tests and comparison of differences in inhibition zones, respectively.

A ratio of the MIC values of the IP to IPI of more or equal to 8 , presence of an extra inhibition zone between IPM and IPI or deformation of the ellipses were interpreted as a positive result of MBLs production.

Amplification of the genes responsible for production of carbapenemases. For DNA isolation a Genomic Mini Kit (A\&A Biotechnology) was used. A single $P$. aeruginosa colony from a 24-hour culture on PseudoSel Agar (Becton-Dickinson) was inoculated into $3 \mathrm{ml}$ of liquid Trypcase-Soy Broth (Becton Dickinson) and incubated for 24 hours at $35^{\circ}$ C. $0.5 \mathrm{ml}$ of overnight culture was used according to the manufacturer's guide. PCR assay was conducted using the method previously described by Pitout et al. (2005) with two pairs of starters for amplification of $b l a_{\mathrm{IMP}}$ and $b l a_{\mathrm{VIM}}$ genes of IMP- and VIM-type MBLs, which amplified 587-bp and 382-bp amplicons, respectively. The composition of the reaction mixture and PCR temperature profile were as previously described (Pitout et al., 2005) with our own modification: lack of initial incubation step at $37^{\circ} \mathrm{C}$ and using Sigma products. PCR products were analyzed by 1-hour electrophoresis with $1.5 \%$ agarose gel in TBE buffer (Tris/Boric Acid/EDTA, Bio-Rad). The gels were stained 40 minutes with ethidium bromide, washed 20 minutes in deionized water, the PCR products were visualized with UV light using Gel Doc 2000 program (Bio-Rad) and compared with positive controls using recorded files in Quantity One 4.1.1 (Bio-Rad).

\section{Results}

Screening for MBLs using phenotypic methods. Double disc synergy tests with ceftazidime and 2-MPA and using IPM with EDTA gave $30(42.9 \%)$ and 
Table II

Comparison of methods for detection of MBLs-producing Pseudomonas aeruginosa strains $(\mathrm{n}=70)$

\begin{tabular}{|l|c|c|}
\hline \multirow{2}{*}{ Method used } & \multicolumn{2}{|c|}{ Positive results } \\
\cline { 2 - 3 } & $\mathrm{n}$ & $\%$ \\
\hline $\begin{array}{l}\text { Double-disc synergy test with imipenem } \\
(10 \mu \mathrm{g}) \text { and 0.5 M EDTA }\end{array}$ & 51 & 72.9 \\
\hline $\begin{array}{l}\text { Comparison of differences in inhibition zones } \\
\text { between imipenem }(10 \mu \mathrm{g}) \text { and imipenem } \\
\text { with EDTA (IPM/EDTA, 10/1000 } \mu \mathrm{g})\end{array}$ & 32 & 45.7 \\
\hline $\begin{array}{l}\text { Double-disc synergy test with ceftazidime } \\
(30 \mu \mathrm{g}) \text { and 2-MPA }\end{array}$ & 30 & 42.9 \\
\hline Etest MBL (AB Biodisk) & 4 & 5.7 \\
\hline $\begin{array}{l}\text { PCR assay for } \text { bla }_{\mathrm{IMP}} \text { and } \text { bla }_{\mathrm{VIM}} \text { genes } \\
\text { responsible for MBLs production }\end{array}$ & 1 & 1.4 \\
\hline
\end{tabular}

$51(72.9 \%)$ positive results, respectively. $32(45.7 \%)$ and $4(5.7 \%)$ strains gave positive results of expressing MBLs using comparison of inhibition zones diameters between IPM and IPI and Etest MBL, respectively (Table II). In case of one strain ratio of the MICs of the IP to IPI was 128 , and in case of the other Etest MBL-positive strains the ratio equaled 8-11.

PCR assay results. Using PCR assay one positive result was obtained - the product of one amplification reaction, illustrated in Fig. 1, represented the same size as positive control possessing bla $_{\mathrm{VIM}}$ gene. In antimicrobial susceptibility testing this strain was resistant to all beta-lactams tested and positive results for detection of MBLs were obtained with all the methods included in this study.

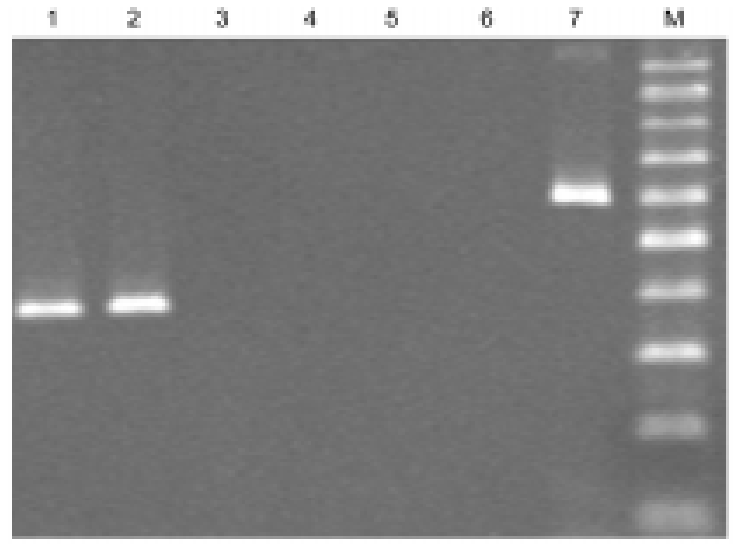

Fig. 1. Gel-electrophoresis of PCR products of examined $P$. aeruginosa strains and controls.

M - DNA size marker 100-1000 bp; lane 1, 7 - positive controls of $b l a_{\mathrm{VIM}}$ and $b l a_{\mathrm{IMP}}$ genes, respectively; lane 6 - negative control; lanes $2-5$ - examined strains.

\section{Discussion}

Among phenotypic methods for MBLs detection (Deptuła and Gospodarek, 2005) The National Reference Centre for Antimicrobial Susceptibility Testing recommends double disc synergy tests in two combinations: ceftazidime (CAZ $30 \mu \mathrm{g}$ ) or imipenem (IPM $10 \mu \mathrm{g}$ ) discs $20 \mathrm{~mm}$ distant to $2-3 \mu \mathrm{l}$ of 2-mercaptopropionic acid or $10 \mu \mathrm{l}$ of $0.5 \mathrm{M}$ EDTA discs (Hryniewicz et al., 2006). This method, when properly performed according to Picão et al. (2008) allows to detect $100 \%$ of $P$. aeruginosa strains producing MBLs (Picão et al., 2008). Efficacy of the test using 2-MPA according to authors is similar to PCR assay detecting $b l a_{\mathrm{IMP}-1}$ genes responsible for IMP-type enzymes (Arakawa et al., 2000). In our results, a difference was observed $-42.9 \%$ versus $1.4 \%$ obtained by PCR assay.

Using double disc synergy tests with imipenem and EDTA the highest amount of positive results in MBLs detection was obtained $-72.9 \%$ of examined strains, suggesting the highest sensitivity of this method, particularly in the case of Pseudomonas spp. strains, according to the authors of this method (Lee et al., 2003).

Using comparison of inhibition zones diameters between IPM/EDA and IPM, $45.7 \%$ of positive results of MBLs detection were obtained, affirming the usefulness of this method in the detection of MBLsproducing $P$. aeruginosa strains and confirming the results obtained by other authors (Hemalatha et al., 2005, Qu et al., 2009, Yong et al., 2002).

Annual Recommendations on Etests MBL from the National Reference Centre for Antimicrobial Susceptibility Testing (Hryniewicz et al., 2006) were also included. Using this method positive results for 5.7\% $P$. aeruginosa strains were obtained. According to the manufacturer's guide and results obtained by other authors (Lee et al., 2005, Walsh et al., 2002), this test has almost $100 \%$ sensitivity and specificity in MBLs detection in $P$. aeruginosa, according to the results using $b l a_{\mathrm{IMP}-1}$ and $b l a_{\mathrm{VIM}-2}$ positive strains. Similar results on low number of strains using tests with imipenem and 2-MPA (Kim et al., 2007), suggest increasing the range of used methods by another test. Yan et al. (2004) claim that Etest MBL has low specificity.

Using PCR assay, the lowest number of positive results of MBLs detection in P. aeruginosa was observed, suggesting different mechanism of resistance to carbapenems than MBLs (i.e. lower production of OprD protein or presence efflux pumps (Quale et al., 2006).

The highest percentage of positive results for detection of MBLs in P. aeruginosa was obtained using double disc synergy tests with imipenem and EDTA, but the specificity of this method may be low.

Using PCR assay, the lowest percent (1.4\%) of positive results for MBLs detection in investigated strains was obtained.

The absence of the searched genes in the carbapenemase resistant strains studied using PCR may indicate the occurrence of other than MBLs-based mechanisms of resistance to carbapenems among these strains. 


\section{Literature}

Arakawa Y., N. Shibata, K. Shibayama, H. Kurokawa, T. Yagi, H. Fujiwara and M. Goto. 2000. Convenient test for screening metallo- $\beta$-lactamase producing Gram-negative bacteria by using thiol compounds. J. Clin. Microbiol. 38: 40-43.

Deptula A. and E. Gospodarek. 2005. Resistance to carbapenems by metallo-beta-lactamases production - detection methods and cost analysis (in Polish). Laboratorium (Special Edition: Microbiology). 2: 19-21.

Fiett J, Baraniak A, Mrówka A, Fleischer M, Drulis-Kawa Z, Naumiuk L, Samet A, Hryniewicz W, Gniadkowski M. 2006. Molecular epidemiology of acquired-metallo-beta-lactamaseproducing bacteria in Poland. Antimicrob. Agents Chemother. 50: 880-886.

Hemalatha V., U. Sekar and V. Kamat. 2005. Detection of metallo beta-lactamase producing Pseudomonas aeruginosa in hospitalized patients. Indian J. Med. Res. 122: 148-152.

Hryniewicz W., A. Sulikowska, K. Szczypa, J. KrzysztofRussjan and M. Gniadkowski. 2006. Recommendations for susceptibility testing of selected bacterial species to antimicrobial agents. Narodowy Instytut Zdrowia Publicznego, Warszawa.

Kim S.Y., S.G. Hong, E.S. Moland and K.S. Thomson. 2007. Convenient test using a combination of chelating agents for detection of metallo- $\beta$-lactamases in the clinical laboratory. J. Clin. Microbiol. 45: 2798-2801.

Lee K., Y.S. Lim, D. Yong, J.H. Yum and Y. Chong. 2003. Evaluation of the Hodge test and the imipenem-EDTA DoubleDisk Synergy Test for differentiating metallo- $\beta$-Lactamaseproducing isolates of Pseudomonas spp. and Acinetobacter spp. J. Clin. Microbiol. 41: 4623-4629.

Lee K., D. Yong, J.H. Yum, Y.S. Lim, A. Bolmström, A. Qwärnström, A. Karlsson and Y. Chong. 2005. Evaluation of Etest MBL for detection of blaIMP-1 and blaVIM-2 allele- positive clinical isolates of Pseudomonas spp. and Acinetobacter spp. J. Clin. Microbiol. 43: 942-944.

Performance Standards for Antimicrobial Susceptibility Testing; Seventeenth Informational Supplement. CLSI M100-S17, Vol. 27, No. 1, 2007.

Picão R.C., S.S. Andrade, A.G. Nicoletti, E.H. Campana, G.C. Moraes, R.E. Mendes and A.C. Gales. 2008. Metallo-betalactamase detection: comparative evaluation of double-disk synergy versus combined disk tests for IMP-, GIM-, SIM-, SPM-, or VIM-producing isolates. J. Clin. Microbiol. 46: 2028-2037.

Pitout J.D., D.B. Gregson, L. Poirel, J.A. McClure, P. Le and D.L. Church. 2005. Detection of Pseudomonas aeruginosa producing metallo- $\beta$-lactamases in a large centralized laboratory. J. Clin. Microbiol. 43: 3129-3135.

Qu T.T., J.L. Zhang, J. Wang, J. Tao, Y.S. Yu, Y.G. Chen, J.Y. Zhou and L.J. Li. 2009. Evaluation of phenotypic tests for the detection of metallo-beta-lactamase-producing Pseudomonas aeruginosa in China. J. Clin. Microbiol. 47: 1136-1142.

Quale J., S. Bratu, J. Gupta and D. Landman. 2006. Interplay of efflux system, ampC, and oprD expression in carbapenem resistance of Pseudomonas aeruginosa clinical isolates. Antimicrob. Agents Chemother. 5: 1633-1641.

Walsh T.R., A. Bolmström, A. Qwärnström and A. Gales. 2002. Evaluation of a new Etest MBL for detecting metallo-betalactamases in routine clinical testing. J. Clin. Microbiol. 40: 2755-2759.

Yan J.J., J.J. Wu, S.H. Tsai and C.L. Chuang. 2004. Comparison of the double-disk, combined disk, and Etest methods for detecting metallo-beta-lactamases in gram-negative bacilli. Diagn Microbiol. Infect. Dis. 49: 5-11.

Yong D., K. Lee, J.H. Yum, H.B. Shin, G.M. Rossolini and Y. Chong. 2002. Imipenem-EDTA disk method for differentiation of metallo- $\beta$-lactamase-producing clinical isolates of Pseudomonas spp. and Acinetobacter spp. J. Clin. Microbiol. 40: 3798-3801. 